\title{
Proposal of a new classification of postoperative ileus based on its clinical impact-results of a global survey and preliminary evaluation in colorectal surgery
}

\author{
Aurélien Venara ${ }^{1,2,3}$ - Karem Slim ${ }^{4}$ - Jean-Marc Regimbeau ${ }^{5}$ - Pablo Ortega-Deballon ${ }^{6}$. \\ Bruno Vielle $^{7} \cdot$ Emilie Lermite $^{1,2}$ • Guillaume Meurette ${ }^{3,8}$ - Antoine Hamy ${ }^{1,2}$ \\ Department of Visceral and Endocrinal Surgery, University Hospital \\ of Angers, 4 rue Larrey, 49933 Cedex 9 Angers, France \\ 2 LUNAM, University of Angers, Angers, France \\ 3 UMR INSERM U1235 - TENS, School of Medicine University of \\ Nantes, 1 rue Gaston Veil, 44035 Nantes, France \\ 4 Department of Visceral Surgery, CHU Estaing, Clermont \\ Ferrand, France \\ $5 \quad$ Visceral and Endocrinal Surgery Department, University Hospital of \\ Amiens, Amiens, France \\ 6 Department of Digestive and Endocrine Surgical Oncology, \\ University Hospital of Dijon, Dijon, France \\ 7 Department of Biostatistics, University Hospital of Angers, \\ Angers, France \\ 8 Visceral and Endocrinal Surgery Department, University Hospital of \\ Nantes, 1 rue Gaston Veil, 44035 Nantes Cedex, France \\ included who underwent colorectal surgery. Forty of them \\ experienced primary postoperative ileus (23.1\%). Grade A \\ occurred in 10 cases, grade B in 10 cases, grade $\mathrm{C}$ in 14 cases, \\ grade D1 in 2 cases, and grade D2 in 2 cases. POI-related \\ death (grade E) occurred in 2 cases. Patients with grade A \\ POI recovered their gastrointestinal function significantly \\ faster than those with higher grades $(p=0.01)$, and were more \\ likely to undergo laparoscopic surgery $(p=0.04)$. The \\ Intraclass Correlation Coefficient (ICC) was 0.83 in the over- \\ all population, and 0.83 and 0.82 respectively in the junior and \\ senior surgeon populations. \\ Conclusion This classification is easy to both use and repro- \\ duce. It will improve the reproducibility, evaluation, and as- \\ sessment of POI. These preliminary results should be con- \\ firmed in a multi-centric international study.
}

\begin{abstract}
Purpose There is no consensual definition of postoperative ileus (POI), which leads to a lack of reproducibility. The aims of this study were (i) to propose and evaluate a classification of postoperative ileus based on its consequences and (ii) to assess the reproducibility of the classification.

Methods A national global survey was carried out according to the DELPHI method in order to create a classification of primary POI. The classification was subsequently tested on a cohort of patients who underwent colorectal surgery. Finally, a reproducibility test was performed in five teaching hospitals with junior and senior surgeons.

Results A five-stage classification was proposed: grade A (least) to grade E (worst). For better differentiation, subcategories (D1/D2) were included. Overall, 173 patients were
\end{abstract}

Keywords Postoperative ileus $\cdot$ Classification $\cdot$ Postoperative morbidity

\section{Background}

In 2005, Kehlet et al. concluded that a clear definition of postoperative ileus (POI) was required [1]. Ten years later, the need for a consensual definition still exists, especially with regard to the cutoff point for normal POI [2]. Nevertheless, literature has become more homogenous since the definition proposed by Vather et al. in 2013 [3] and the confirmation of the endpoints by Van Bree et al. in 2014 [4]. However, there is a lack of agreement about the cutoff point for normal or prolonged POI. Postoperative ileus is reported to occur in $3-$ $32 \%$ of cases in overall surgery [2, 5-10]. However, cutoff points vary between 3 and 7 days [3, 5-9], inevitably changing the POI rate within the same population [11]. This leads to non-reproducibility of studies. The hope to answer this 
question of delay is probably futile. Indeed, knowledge of physiopathology is limited and unlikely to provide answers due to inter-individual variability. Vagal activation is one of the pathways that resolve POI [12]. The pathogenesis of POI is explained by a reactive inflammation after intestinal manipulation [13]. First effects occur within $3 \mathrm{~h}$ after operation. However, the delay before vagal activation and the pathway leading to vagal activation are not known.

Definitions from Vather et al. [3] and Van Bree et al. [4] are meaningful in clinical practice because they help with the diagnosis of POI. However, in case of clinical studies, these definitions remain insufficient due to the lack of consensus regarding the normal delay to the recovery of gastrointestinal functions. Drawing on the model of the Clavien-Dindo classification [14], POI has several consequences that could be used to put forward a classification that would be meaningful in clinical studies and could ensure better reproducibility between works. Indeed, PPOI (prolonged postoperative ileus) increases not only the length of hospital stay but also the management costs [15], the risk of severe complications, and the need for further surgery [16]. Consequences of POI include vomiting, which can lead to pulmonary aspiration [17]. POI also causes impaired fluid, electrolyte and nutrient reabsorption, fluid and electrolyte imbalance, and nutritional deficiencies [17].

When postoperative ileus is due to an external cause of the bowel, POI is classed as a secondary ileus. It differs from primary POI in that it does not respond to the same physiopathology. Secondary POI is the consequence of an adverse effect of surgery [15], whereas primary POI is an autonomous paralysis of the smooth muscle of the bowel that has its own consequences. Given this difference in physiopathology, primary POI should have its own specific classification that excludes secondary POI.

The main aim of this work was to propose a new classification of primary POI based on its consequences and the possibility to use it in clinical and experimental studies. Additional goals were to evaluate the classification in a preliminary study that included a cohort of patients undergoing colorectal surgery and to assess its reproducibility through a national survey.

\section{Materials and methods}

All steps are summarized in Fig. 1.

\section{Proposal of a classification-National DELPHI survey}

A work group of surgeons and anesthetists involved in early rehabilitation was created. Twenty-two contributors from France, Belgium, and Switzerland participated in the survey. They were all employed in high-volume centers and were involved in improving the postoperative course. The method used to create the classification was the DELPHI method [18].

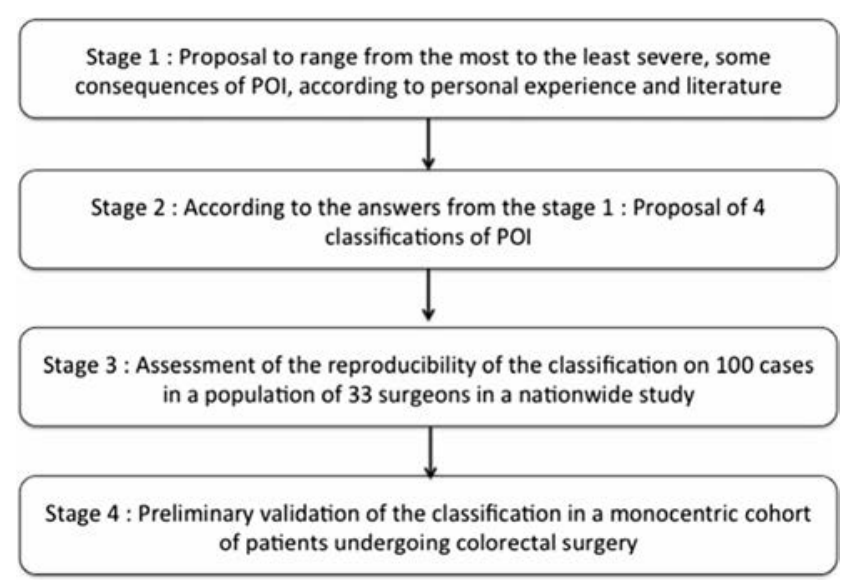

Fig. 1 Flowchart of the different stages of the classification proposal

Contributors were contacted by e-mail. The first e-mail asked participants to classify, from most severe to least severe, the different consequences of POI. A space was left free to allow participants to suggest other items. The survey was performed using the website SurveyMonkey®. The items and results of the first stage of this work are reported in Fig. 2. The results of the survey are noted according to the valorization of the website (number of citations, range of citations, etc.).

The items were proposed according to literature and the personal experience of the investigators. If participants did not answer the e-mail, a reminder was sent 2 weeks later to request an answer.

For the second stage of this work, four classifications were proposed according to the answers received. A new e-mail was sent to the participants. They were asked to select the classification that was, in their opinion, best adapted for POI. Sixteen contributors responded.

Forty-five percent of the contributors agreed on the same classification. The classification was subsequently adapted to suit all the contributors.

\section{Validation of the classification}

The classification was assessed on a cohort of 173 consecutive patients who underwent colorectal surgery between 1 June 2012 and 1 July 2014 in a single tertiary center. Data was retrospectively extracted from a prospective database, collected with the aim of performing an audit of enhanced recovery protocols. All consecutive patients who underwent emergency surgery or laparotomy were also included. Some of these patients were included in a previous study in which we demonstrated that enhanced recovery programs can reduce POI [11].

In this monocentric cohort, patients who required intensive care following surgery were not included as they were unable to follow the early rehabilitation protocol.

Information regarding the perioperative course was collected in an anonymized electronic database and related to the postoperative course: occurrence of POI, need for prokinetics, 
Fig. 2 Bar chart of the results of the first stage of the survey

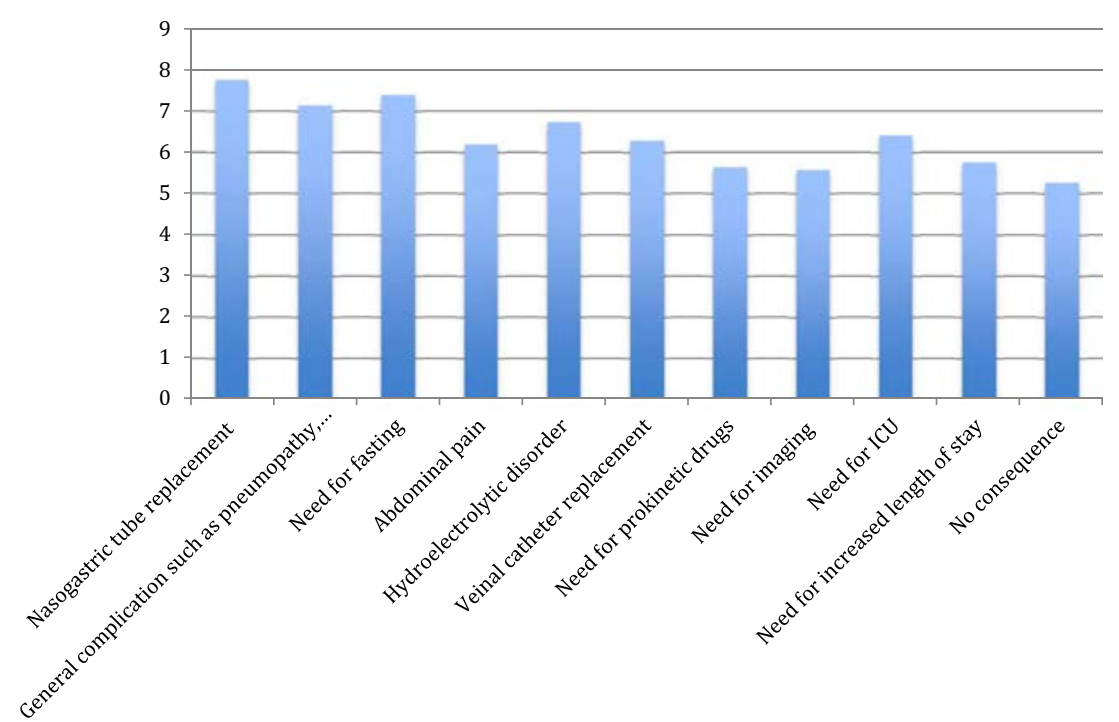

nasogastric tube, vein access, need for radiological imaging, general complications, need for further surgery, length of stay, and time for bowel motility recovery.

\section{Reproducibility of the classification}

A questionnaire was sent to five heads of surgical departments in university hospitals in France. Three of them were requested to transmit the questionnaire to five senior surgeons (MD or $\mathrm{MD}-\mathrm{PhD}$ ) and five junior surgeons. The number of observers was decided according to the calculation of the population (see below). After 2 weeks, the heads of units were sent a reminder e-mail. After the second deadline, 33 surgeons had answered the questionnaire (16 junior surgeons and 17 senior surgeons). Junior surgeons were defined as residents whereas senior surgeons were defined as all graduated medical doctors.

The questionnaire presented 101 fictitious cases of patients who experienced POI. The aim was to classify the POI according to the classification.

\section{Definitions}

Length of stay (LOS) was the theoretical LOS. It was defined as the first postoperative day (POD) on which the patient met all the criteria for discharge. The patient met all the criteria for discharge when he was able to independently perform usual daily activities. Criteria retained were the same in case of POI.

POI was considered since the first POD. The definition from Vather et al. for PPOI was retained, and POI was linked to $\geq 2$ of the five following criteria [3]:

- Nausea or vomiting over the preceding $12 \mathrm{~h}$

- Inability to tolerate a solid or semisolid oral diet over the preceding two mealtimes
- Abdominal distension

- Absence of flatus and stool over the preceding $24 \mathrm{~h}$

- Radiological evidence of ileus on abdominal plain film or $\mathrm{CT}$ over the preceding $24 \mathrm{~h}$

Gastrointestinal motility recovery was defined as the association of solid food tolerance and stool exoneration [4]. We considered solid food tolerance as the moment when a patient was able to eat half of a standard hospital meal.

Secondary POI was defined as reported by Asgeirsson et al. [15]. Secondary POI was considered if it was associated with intra-abdominal complications. Secondary POI could be characterized after the diagnosis of POI, during a CT scan. CT scans were performed in the event of prolonged POI or rising inflammatory syndrome.

The study was approved by the local ethical committee.

\section{Statistical analysis}

- Calculation of the effectives

To reach an ICC of 0.8 (width of $95 \%$; confidence inter$\mathrm{val}=0.1$ ), the required enrolment was calculated: 12 surgeons were needed for 90 observations performed. Due to the risk of incomplete data (10\%), 101 observations were proposed.

\section{- Statistical tests}

Values are expressed as median (range) or mean ( \pm standard deviation).

The correlation between the ileus grade according to the classification and the delay of stool recovery, gas recovery, and the length of stay was assessed using linear regression.

Concordance between the different surgeons was assessed using the Intraclass Correlation Coefficient (ICC). 
Type 1 error was set at 0.05 . Statistics were completed using the program Statistical Package for the Social Sciences (SPSS version 10.0. Chicago, IL). The value $p<0.05$ was considered statistically significant.

\section{Results}

\section{Proposal of a classification}

After 2 months, the DELPHI survey was completed, and the following classification was put forward and accepted by most participants. This classification concerned all adverse effects exclusively relating to POI.

- Grade A: no consequence of POI apart from an increase in the length of stay.

- Grade B: need for symptomatic measures or diagnostic examinations (such as laxatives, prokinetic drugs, antispasmodic drugs, antiemetic drugs, need for decreased regimen, or need for vein access, etc.).

- Grade C: need for nasogastric tube intake or hospital readmission after discharge.

- In the event where the nasogastric tube is not removed immediately, grade $\mathrm{C}$ should only be considered if the nasogastric tube was removed before it required replacement.

- Grade D: severe consequences of POI.

- D1: general complications (such as ionic imbalance, pneumopathy, auricular fibrillation, etc.)

- D2: need for intensive care unit or further surgery.

- Grade E: death.

\section{Assessment of the reproducibility}

The ICC index was 0.82 in the overall population (CI95\% $0.77-0.86)$.

In the junior surgeon population, the ICC index was 0.82 (CI95\% 0.78-0.86), whereas it was 0.81 (CI95\% 0.77-0.85) in the senior surgeon population.

\section{Validation of the classification}

Among the 173 patients included, ileus occurred in 50 patients (28.9\%). Ten patients experienced secondary ileus due to anastomotic leakage or hemoperitoneum.

Forty patients experienced primary ileus (23.1\%). The classification was tested exclusively on primary POI. Grade A occurred in 10 cases (25\%), grade B in 10 cases $(25 \%)$, grade
$\mathrm{C}$ in 14 cases (35\%), grade D1 in 2 cases (5\%), grade D2 in 2 cases $(5 \%)$, and grade $\mathrm{E}$ in 2 cases $(5 \%)$.

Median delay to first flatus was 3 days $(2 ; 3)$ and median delay to first stool was 4 days $(4 ; 5)$ in the overall population. Median length of stay was 9 days $(7 ; 12)$.

According to this classification, laparoscopy was associated with a smaller grade of POI (grade A; $p=0.04$ ) (Table 1). Conversely, a higher ASA score was associated with a higher rate of high-grade POI ( $p=0.049$ and $p=0.01)$.

Time to recovery of gastrointestinal motility (solid food tolerance + stool) was not correlated with the grade of our classification of POI $\left(r^{2}=0.01 ; p=0.54\right)$ in linear regression, but POI grade A had a significantly faster recovery of gastrointestinal motility $(p=0.01)$.

\section{Discussion}

Although there is now a definition for POI, the absence of a consensus regarding the cutoff point for normal POI within the surgical community leads to a lack of reproducibility of clinical studies. A classification based on objective consequences of POI could make studies on POI reliable. Indeed, in five recent studies analyzing risk factors for POI, four different cutoff points for POI were used [5-7, 9, 19]. In these studies, the risk factors and the POI rate were different. Some studies considered that patients suffered from ileus if they required a nasogastric tube [20]. The proposed classification showed that, in our series, only 14 patients (35\%) required a nasogastric tube, which highlights the need for a consensual classification based on consequences.

Various factors, such as constipation, influence the time to bowel motility recovery, which leads to a possible misinterpretation of these factors as risk factors for POI [21]. As such, we believe that a classification of POI should be based on consequences rather than delay. The definition of PPOI proposed by Vather et al. is clear [3] and should be extended to all POI, without including the notion of delay. Indeed, in our experience, some patients treated through early recovery protocols did not experience gastrointestinal blockade. That is why we think that considering POI from the first day could ensure better reproducibility of POI incidence between studies. In addition, the delay is too random. Despite knowledge of some classifiers in the series of events that lead to POI and its resolution, none of the classifiers make it possible to predict the duration of normal POI.

Interestingly, in our cohort, the grades of this classification were not correlated with the duration of POI. However, known risk factors of ileus, such as respiratory comorbidities or preoperatory sepsis (which increase the ASA score) [5, 6], were associated with high POI severity, while laparoscopy was associated with a lower grade of POI severity. Laparoscopy is known to be protective against POI [7]. 
Table 1 Association between the grade of POI and some of the different recognized factors of POI in literature

\begin{tabular}{|c|c|c|c|c|c|c|c|c|c|}
\hline & \multicolumn{3}{|l|}{ Grade A } & \multicolumn{3}{|l|}{ Grade B } & \multirow[b]{2}{*}{ Yes $n=14$} & \multicolumn{2}{|l|}{ Grade C } \\
\hline & Yes $n=10$ & No $n=30$ & $p$ value & Yes $n=10$ & No $n=30$ & $p$ value & & No $n=26$ & $p$ value \\
\hline Gender, male & $6(60 \%)$ & $19(63.3 \%)$ & $>0.9$ & $4(40 \%)$ & $21(70 \%)$ & 0.13 & $12(46.2 \%)$ & $14(53.8 \%)$ & 0.12 \\
\hline Age, years & $55(68 ; 75)$ & $80(67 ; 85)$ & 0.06 & $82(56 ; 88)$ & $81(67 ; 85)$ & 0.38 & $73(67 ; 87)$ & $3(2 ; 3)$ & 0.57 \\
\hline BMI, $\mathrm{kg} / \mathrm{m}^{2}$ & $26(20 ; 28)$ & $23(24 ; 27)$ & 0.68 & $26(20 ; 28)$ & $24.5(23 ; 27)$ & 0.31 & $25.5(24 ; 27)$ & $24(22 ; 27)$ & 0.17 \\
\hline $\begin{array}{l}\text { Duration of surgical } \\
\text { procedure }\end{array}$ & $180(180 ; 240)$ & $150(120 ; 210)$ & 0.25 & $180(180 ; 240)$ & $150(120 ; 210)$ & 0.34 & $165(145 ; 250)$ & $180(120 ; 213)$ & 0.48 \\
\hline Laparoscopy & $6(60 \%)$ & $6(20 \%)$ & 0.04 & $1(10 \%)$ & $11(36.7 \%)$ & 0.23 & $4(40 \%)$ & $8(66.7 \%)$ & $>0.9$ \\
\hline Manual anastomosis & $6(60 \%)$ & $18(60 \%)$ & $>0.9$ & $8(80 \%)$ & $16(53.3 \%)$ & 0.26 & $6(60 \%)$ & $18(69.2 \%)$ & 0.18 \\
\hline Ostomy & 0 & $1(3.3 \%)$ & $>0.9$ & 0 & $1(3.3 \%)$ & $>0.9$ & 0 & $1(7.1 \%)$ & 0.35 \\
\hline ASA score & & & 0.37 & & & 0.049 & & & 0.014 \\
\hline ASA 1 & $1(10 \%)$ & $2(6.7 \%)$ & & $1(10 \%)$ & $2(6.7 \%)$ & & $1(7.1 \%)$ & $2(7.7 \%)$ & \\
\hline ASA 2 & $6(60 \%)$ & $10(33.3 \%)$ & & $6(60 \%)$ & $10(33.3 \%)$ & & $2(14.3 \%)$ & $14(53.8 \%)$ & \\
\hline ASA 3 & $3(30 \%)$ & $15(50 \%)$ & & $1(10 \%)$ & $17(56.7 \%)$ & & $11(78.6 \%)$ & $7(26.9 \%)$ & \\
\hline ASA 4 & 0 & $3(10 \%)$ & & $2(20 \%)$ & $1(3.3 \%)$ & & 0 & $3(11.5 \%)$ & \\
\hline Emergency surgery & 0 & $4(13.3 \%)$ & 0.55 & $2(20 \%)$ & $2(6.7 \%)$ & 0.22 & $1(7.1 \%)$ & $3(11.5 \%)$ & $>0.9$ \\
\hline $\begin{array}{l}\text { Indication for colonic } \\
\text { obstruction }\end{array}$ & 0 & $5(16.7 \%)$ & 0.31 & $2(20 \%)$ & $3(10 \%)$ & 0.58 & $1(7.1 \%)$ & $4(15.4 \%)$ & 0.64 \\
\hline Corticotherapy & $1(10 \%)$ & $1(3.3 \%)$ & 0.40 & 0 & $2(6.7 \%)$ & $>0.9$ & $1(3.8 \%)$ & $1(3.8 \%)$ & $>0.9$ \\
\hline Preoperative irradiation & 0 & $1(3.3 \%)$ & $>0.9$ & 0 & $1(3.3 \%)$ & $>0.9$ & $1(7.1 \%)$ & 0 & 0.35 \\
\hline Right colectomy & $5(50 \%)$ & $17(56.7 \%)$ & 0.73 & $7(70 \%)$ & $15(50 \%)$ & 0.46 & $7(50 \%)$ & $15(57.7 \%)$ & 0.64 \\
\hline Length of stay, days & $7(5 ; 8)$ & $10(8 ; 14)$ & $<0.01$ & $10(6 ; 12)$ & $9(8 ; 12)$ & 0.75 & $10(9 ; 14)$ & $8(6 ; 10)$ & 0.03 \\
\hline $\begin{array}{l}\text { Time to GI motility } \\
\text { recovery, days }\end{array}$ & $4(4 ; 4)$ & $5(4.25 ; 6)$ & 0.01 & $5(4.25 ; 5.75)$ & $5(4 ; 5)$ & 0.31 & $5(5 ; 6)$ & $4.5(4 ; 5)$ & 0.13 \\
\hline Time to first flatus, days & $4(4 ; 4)$ & $4(3 ; 5)$ & 0.30 & $5(4.25 ; 5.75)$ & $4(3 ; 5)$ & 0.99 & $4(3 ; 5)$ & $4(4 ; 5)$ & 0.29 \\
\hline Time to first stool, days & $3(2 ; 3)$ & $3(2 ; 4)$ & 0.82 & $3(3 ; 3)$ & $3(2 ; 4)$ & 0.01 & $3.5(2 ; 4)$ & $3(2 ; 3)$ & 0.36 \\
\hline
\end{tabular}

Another interesting result was that while time to GI motility recovery was significantly different between grade A POI and the other grades $(p=0.01)$, there was no significant correlation between POI duration and POI grade according to our classification. In addition, there was a significant difference in the length of hospital stay between grade A POI and the other grades $(p<0.01)$. These results are promising and could lead to better management of POI. However, they should be confirmed in a larger prospective cohort.

Apart from simply classifying POI, another benefit of this classification proposal is the possibility to compare the effectiveness of early rehabilitation protocols in reducing complications that result directly from POI. The effect of protocols on bowel motility remains uncertain, but they seem to improve the delay to bowel motility recovery [22-24]. In addition, there is no information about the reduction of complicated POI. Arguably, in terms of cost-effectiveness and well-being, reducing severe complications of POI is more important for patients and society, rather than gaining 1 day of delay to gas recovery. The proposed classification would make it possible to assess early rehabilitations protocols.

Finally, the reproducibility of the proposed classification was assessed in a population of 33 surgeons (17 senior surgeons and 16 junior surgeons) and the overall ICC index was 0.85 . Literature and statistical analyzers consider this result as indicative of good reproducibility [25].

It is likely that this result is underestimated, given that surgeons assessed 101 cases of patients with POI at the same time, which may have resulted in a lack of concentration towards the end of the test.

In addition, another factor resulting in a decrease of the ICC index was the confusion surrounding the Clavien-Dindo classification [14]. Several surgeons classed deaths caused by general complications as POI-related. To improve understanding of the classification and ensure better reproducibility, the classification has been made more detailed in the final version above. Any confusion should disappear with experience and use, and should be assessed further.

As a general rule, data is usually collected by junior surgeons, which is why they were specifically included in this work, which is aimed at assessing reproducibility. Junior surgeons found reproducibility to be good and the ICC index was 0.82 . This result was the same as that of senior surgeons, which support the argument that the proposed classification is easy to use. 
The classification's potential limitations are the same as those of the Clavien-Dindo classification [14]. The classification can mainly be used for clinical or experimental studies; it has a low significance in usual practice given that it does not lead to a change in management. However, better reproducibility between studies will lead to a better understanding of pathways that lead to POI according to the severity of its consequences. A better understanding of POI will, in turn, lead to better management in clinical practice.

Limitations also include subjectivity of the management and subjectivity of the interpretation. However, reproducibility between multiple teams of surgeons with different levels of training and habits was acceptable and comparable with the one displayed by Dindo et al. [14], who reported an $87-91 \%$ accuracy rate at the beginning of their experiment. Moreover, this classification reflects the management of POI and could allow for a better comparison and understanding of management between teams. In fact, management is subjective but the classification is objective, based on the reality of management.

We have shown that the proposed classification is easy to use by any surgeon of any level. It could be the first stage before the acceptance of a consensual classification of POI, which would lead to a better comprehension of this frequent postsurgical complication and, subsequently, to more effective management in clinical practice.

\section{Conclusion}

The proposal of a POI classification based on its consequences is the result of the French national survey DELPHI. The classification is easy to use and reproduce by both junior and senior surgeons. A general use of this classification in literature on POI will improve the reproducibility and evaluation of POI between different studies. Given that the classification was validated by a preliminary study on a cohort of patients who underwent colorectal surgery only, an international multicentric study should confirm these results among the whole postoperative course of every surgery.

Acknowledgements The authors would like to acknowledge all the surgeons and anesthetists who participated in the global survey and were involved in the assessment of the reproducibility of the classification: Dr Mucci S, Dr Casa C, Dr Fuks D, Dr Hardwigsen J, Dr Gaujoux S, Pr Pocard M, Dr Levard H, Pr Sastre B, Pr Dousset B, Dr Mariani P, Pr Lehur P, Dr Rio D, Pr Fingerhut A, Dr Rault A, Dr Finel JB, Dr Branger F, Dr Le Naoures P, Dr Blanchard L, Pr Sabbagh C, Mr Jaouen R, Mrs Peneau C, Mr Colas PA, Mr Barbieux J, Mr Mougin J, Mr Le Fouler A, Dr Bouvier A, Dr Landreau P, Mr Gilbert A, Mr Borraccino B, Mr Gout M, Dr Santucci N, Mr Khaoudy I, Mr Gracient A, Mr Prevot F, Mr Chaibi S, Mr Mariage M, Dr Dhari A, and Mr Darmon I.

This list is unfortunately incomplete but a part of the survey was anonymous to avoid bias. The authors would also like to acknowledge the participants who did not give their name.

\section{Compliance with ethical standards}

Conflict of interest The authors declare that they have no conflict of interest.

\section{References}

1. Kehlet H, Williamson R, Büchler MW, Beart RW (2005) A survey of perceptions and attitudes among European surgeons towards the clinical impact and management of postoperative ileus. Colorectal Dis 7:245-250

2. Wolthuis AM, Bislenghi G, Fieuws S, et al (2015) Incidence of prolonged postoperative ileus after colorectal surgery: a systematic review and meta-analysis. Colorectal Dis

3. Vather R, Trivedi S, Bissett I (2013) Defining postoperative ileus: results of a systematic review and global survey. J Gastrointest Surg 17:962-972

4. Van Bree SHW, Bemelman WA, Hollmann MW et al (2014) Identification of clinical outcome measures for recovery of gastrointestinal motility in postoperative ileus. Ann Surg 259:708-714

5. Chapuis PH, Bokey L, Keshava A et al (2013) Risk factors for prolonged ileus after resection of colorectal cancer: an observational study of 2400 consecutive patients. Ann Surg 257:909-915

6. Moghadamyeghaneh Z, Hwang GS, Hanna MH, et al (2015) Risk factors for prolonged ileus following colon surgery. Surg Endosc

7. Vather R, Josephson R, Jaung R et al (2015) Development of a risk stratification system for the occurrence of prolonged postoperative ileus after colorectal surgery: a prospective risk factor analysis. Surgery 157:764-773

8. Artinyan A, Nunoo-Mensah JW, Balasubramaniam S et al (2008) Prolonged postoperative ileus-definition. risk factors. and predictors after surgery. World $J$ Surg 32:1495-1500

9. Millan M, Biondo S, Fraccalvieri D et al (2012) Risk factors for prolonged postoperative ileus after colorectal cancer surgery. World $J$ Surg 36:179-185

10. Vather R, Josephson R, Jaung R et al (2015) Gastrografin in prolonged postoperative ileus: a double-blinded randomized controlled trial. Ann Surg 262:23-30

11. Barbieux J, Hamy A, Talbot MF, et al (2016) Does early rehabilitation reduce the time to bowel motility recovery after colorectal surgery? J Visc Surg.

12. de Jonge WJ, van der Zanden EP, The FO et al (2005) Stimulation of the vagus nerve attenuates macrophage activation by activating the Jak2-STAT3 signaling pathway. Nat Immunol 6:844-851

13. Kalff JC, Carlos TM, Schraut WH et al (1999) Surgically induced leukocytic infiltrates within the rat intestinal muscularis mediate postoperative ileus. Gastroenterology 117:378-387

14. Dindo D, Demartines N, Clavien P-A (2004) Classification of surgical complications: a new proposal with evaluation in a cohort of 6336 patients and results of a survey. Ann Surg 240:205-213

15. Asgeirsson T, El-Badawi KI, Mahmood A et al (2010) Postoperative ileus: it costs more than you expect. $\mathrm{J} \mathrm{Am} \mathrm{Coll}$ Surg 210:228-231

16. Tevis SE, Carchman EH, Foley EF et al (2015) Postoperative ileus - more than just prolonged length of stay? J Gastrointest Surg 19:1684-1690

17. Bragg D, El-Sharkawy AM, Psaltis E et al (2015) Postoperative ileus: recent developments in pathophysiology and management. Clin Nutr 34:367-376

18. Milholland AV, Wheeler SG, Heieck JJ (1973) Medical assessment by a Delphi group opinion technic. N Engl J Med 288:1272-1275 
19. Vather R, Bissett IP (2013) Risk factors for the development of prolonged post-operative ileus following elective colorectal surgery. Int J Colorectal Dis 28:1385-1391

20. Kronberg U, Kiran RP, Soliman MSM et al (2011) A characterization of factors determining postoperative ileus after laparoscopic colectomy enables the generation of a novel predictive score. Ann Surg 253:78-81

21. Lee TH, Lee JS, Hong SJ et al (2015) Risk factors for postoperative ileus following orthopedic surgery: the role of chronic constipation. J Neurogastroenterol Motil 21:121-125

22. Basse L, Raskov HH, Hjort Jakobsen D et al (2002) Accelerated postoperative recovery programme after colonic resection improves physical performance. pulmonary function and body composition. Br J Surg 89:446-453

23. Poon JTC, Fan JKM, Lo OSH, Law WL (2011) Enhanced recovery program in laparoscopic colectomy for cancer. Int J Colorectal Dis 26:71-77

24. Spanjersberg WR, Reurings J, Keus F, van Laarhoven CJ (2011) Fast track surgery versus conventional recovery strategies for colorectal surgery. Cochrane Database Syst Rev 2:CD007635

25. Fleiss JL (1986) The design and analysis of clinical experiments. Wiley, New York, pp 1-31 